\title{
Deutscher Bibliotheksverband und die Deutsche Telekom Stiftung zeichnen vorbildliche Bibliotheken 2020 aus
}

\author{
Stadtbibliothek Gotha erhält den Preis „Bibliothek des \\ Jahres 2020 in kleinen Kommunen und Regionen“. TIB - \\ Leibniz-Informationszentrum Technik und Naturwissenschaften \\ und Universitätsbibliothek wird „Bibliothek des Jahres 2020“
}

https://doi.org/10.1515/bd-2020-0077

In diesem Jahr zeichnen der Deutsche Bibliotheksverband (dbv) und die Deutsche Telekom Stiftung gleich zwei herausragende Bibliotheken in Deutschland aus: Den mit 20.000 Euro dotierten nationalen Bibliothekspreis erhält in diesem Jahr die „TIB - Leibniz-Informationszentrum Technik und Naturwissenschaften und Universitätsbibliothek“ in Hannover. Der Preis wird der TIB verliehen für ihre herausragende Bibliotheksarbeit im Bereich der strategischen Open ScienceTransformation, Open Access, Forschungsdaten und der digitalen Langzeitarchivierung sowie für ihre exzellenten Entwicklungen von Services an der Schnittstelle von analogen und digitalen Formaten.

Erstmals wird in diesem Jahr auch die Auszeichnung „Bibliothek des Jahres 2020 in kleinen Kommunen und Regionen“ verliehen. Diese Auszeichnung erhält die Stadtbibliothek Gotha. Die Bibliothek in Gotha mit ihren 46.000 Einwohner^innen zeichnet sich durch ihre hervorragende, auf sehr unterschiedliche Zielgruppen abgestimmte Bildungs- und Medienarbeit aus. Dazu gehören u.a. das modular aufgebaute Kinder- und Jugendprojekt „Literatur und Zeitgeschichte“, das Kinderprojekt „Vorlesen an ungewöhnlichen Orten“, die Seniorenakademie, die Erzählcafés sowie die Kinder-Uni in Kooperation mit Museumslöwen e.V. Darüber hinaus ist die Bibliothek Sitz des Kinder- und Jugendforums als Gremium für die kommunale Beteiligung von Kindern und Jugendlichen in der Stadt Gotha.

Dr. Frank Mentrup, Präsident des Deutschen Bibliotheksverbandes und Juryvorsitzender, sagt: „Mit der Auszeichnung ,Bibliothek des Jahres‘ zeichnen wir in diesem Jahr eine wissenschaftliche Bibliothek aus, die eine Vorreiterrolle in der Gestaltung des digitalen Wandels von Bibliotheken einnimmt. Mit ihrer strategischen in die Zukunft gerichteten Bibliotheksarbeit, mit ihrem vielfältigen sozialen und kulturellen Engagement in die Gesellschaft und in die Region hinein, um Wissenschaft und Forschung, Salon-fähig‘ zu machen, und nicht zuletzt durch 
die Aufrechterhaltung der Literaturversorgung durch die TIB-Dokumentlieferung in der Corona-Krise ist die ,TIB - Leibniz-Informationszentrum Technik und Naturwissenschaften und Universitätsbibliothek` eine überaus preiswürdige Bibliothek.“

Und Dr. Ekkehard Winter, Geschäftsführer Deutsche Telekom Stiftung und Mitglied der Jury, ergänzt: „Ich freue mich, dass wir in diesem Jahr gleich zwei Bibliotheken auszeichnen und damit auch das großartige Engagement kleinerer Stadt- und Gemeindebibliotheken sichtbar machen. Die Stadtbibliothek Gotha zeichnet sich durch ihre vielseitige Bildungs- und Medienarbeit, wie die KinderUni, die Seniorenakademie oder ihre Erzählcafés, aus. Damit ist sie ein wichtiger kultureller und gesellschaftlicher Bildungsort für Gotha und seine Umgebung.“

Der Jury gehören Vertreter^innen des Deutschen Bibliotheksverbandes, der Deutsche Telekom Stiftung, des Bundesministeriums für Bildung und Forschung, der Kultusministerkonferenz, des Deutschen Städtetages sowie von Bibliothek \& Information Deutschland (BID) an. Sie entschieden in diesem Jahr über insgesamt 34 Einreichungen.

Die Preisverleihung findet am 24. Oktober 2020 statt.

Nähere Informationen zu den Preisträgern unter: https://www.bibliotheks verband.de/dbv/auszeichnungen/bibliothek-desjahres/preistraeger/2020.html.

\section{Auszeichnung Bibliothek des Jahres}

Die Auszeichnung „Bibliothek des Jahres“ ist der einzige nationale Bibliothekspreis in Deutschland und wird gemeinsam vom Deutschen Bibliotheksverband und der Deutsche Telekom Stiftung vergeben. Mit dieser Auszeichnung soll ein Beitrag zum Image der Bibliotheken in der digitalen Welt geleistet werden. Der Preis ist mit 20.000 Euro dotiert. Die Auszeichnung würdigt Bibliotheken aller Sparten und Größen. Erstmals wird 2020 zusätzlich eine Bibliothek in einer Kommune oder Region mit bis zu 50.000 Einwohnerinnen ausgezeichnet. Der Preis ist mit 7.000 Euro dotiert.

Mehr Informationen unter: https://www.bibliotheksverband.de/dbv/aus zeichnungen/bibliothek-des-jahres.html.

\section{Der Deutsche Bibliotheksverband e.V. (dbv)}

Der Deutsche Bibliotheksverband e.V. (dbv) vertritt mit seinen mehr als 2.100 Mitgliedern bundesweit rund 10.000 Bibliotheken mit 25.000 Beschäftigten und 11 Mio. Nutzer^innen. Sein zentrales Anliegen ist es, Bibliotheken zu stärken, damit sie allen Bürger^innen freien Zugang zu Informationen ermöglichen. Der Verband setzt sich ein für die Entwicklung innovativer Bibliotheksleistungen für Wissenschaft und Gesellschaft. Als politische Interessensvertretung unterstützt der dbv die Bibliotheken insbesondere auf den Feldern Informationskompetenz 
und Medienbildung, Leseförderung und bei der Ermöglichung kultureller und gesellschaftlicher Teilhabe für alle Bürger^innen.

www.bibliotheksverband.de

\section{Die Deutsche Telekom Stiftung}

Die Deutsche Telekom Stiftung wurde 2003 gegründet, um den Bildungs-, Forschungs- und Technologiestandort Deutschland zu stärken. Mit einem Kapital von 150 Millionen Euro gehört sie zu den großen Unternehmensstiftungen in Deutschland. Sie konzipiert und realisiert Projekte, die sich mit Themen aus dem mathematisch-naturwissenschaftlich-technischen Umfeld beschäftigen. Die Stiftung setzt sich dafür ein, dass junge Menschen in einem Bildungs-Ökosystem lernen und initiiert und fördert daher Kooperationen verschiedener Lernorte.

www.telekom-stiftung.de

\section{Pressekontakte}

Kristin Bäßler

Deutscher Bibliotheksverband

Leitung Kommunikation / Pressesprecherin

Tel.: +49 (0)30644989925

E-Mail: baessler@bibliotheksverband.de

Stefanie Thate

Deutsche Telekom Stiftung

Referentin Kommunikation

Tel.: +49 (0)171 8184923

E-Mail: stefanie.thate@telekom-stiftung.de

Dr. Sandra Niemeyer

TIB - Leibniz-Informationszentrum Technik und Naturwissenschaften

und Universitätsbibliothek

Tel.: +49(0)511 7622772

E-Mail: sandra.niemeyer@tib.eu

Nicole Strohrmann

Stadtbibliothek Gotha

Leiterin

Tel.: +49 (0)15140104379

E-Mail: strohrmann.bibliothek@gotha.de 